\title{
Twin-emitter design strategy for use in solution-processable thermally activated delayed fluorescence organic light- emitting diodes
}

Ettore Crovini, ${ }^{\ddagger a}$ Zhen Zhang, ${ }^{\ddagger b}$ Yu Kusakabe, ${ }^{c}$ Yongxia Ren, ${ }^{c}$ Yoshimasa Wada, ${ }^{c}$ Bilal A. Naqvi, ${ }^{d}$ Prakhar Sahay, ${ }^{d}$ Wolfgang Brütting, ${ }^{,}$Katsuaki Suzuki, ${ }^{c}$ Hironori Kaji, ${ }^{* c}$ Stefan Bräse*b,e and Eli Zysman-Colman*a

${ }^{a}$ Organic Semiconductor Centre, EaStCHEM School of Chemistry, University of St Andrews, St Andrews, Fife, KY16 9ST, UK. E-mail: eli.zysman-colman@st-andrews.ac.uk; Web: http://www.zysman-colman.com; Tel: $+44(0) 1334463826$

${ }^{b}$ Institute of Organic Chemistry, Karlsruhe Institute of Technology (KIT), Fritz-Haber-Weg 6, 76131 Karlsruhe, Germany. E-mail: braese@kit.edu; Fax: (+49)-721-6084-8581; Tel: (+49)-721-6084-2903

${ }^{c}$ Institute for Chemical Research, Kyoto University, Uji, Kyoto 611-0011, Japan. E-mail: kaji@scl.kyotou.ac.jp

${ }^{d}$ Experimental Physics IV, Institute of Physics, University of Augsburg, Universitätstrasse. 1, 86159 Augsburg, Germany.

${ }^{e}$ Institute of Biological and Chemical Systems - Functional Molecular Systems (IBCS-FMS), Karlsruhe Institute of Technology (KIT), Hermann-von-Helmholtz-Platz 1, D-76344 Eggenstein-Leopoldshafen, Germany

‡ Ettore Crovini and Zhen Zhang contributed equally to this work.

\section{Abstract}

In this work we showcase the emitter DICzTRZ in which we employed a twin-emitter design of our previously reported material, ICzTRZ. This new system presented a redshifted emission at $488 \mathrm{~nm}$ compared to that of ICzTRZ at $475 \mathrm{~nm}$ and showed a comparable photoluminescence quantum yield of $57.1 \%$ in a $20 \mathrm{wt} \% \mathrm{CzSi}$ film versus 63.3\% for ICzTRZ. The emitter was then incorporated within a solution-processed organic light-emitting diode that showed a maximum external quantum efficiency of $8 \%$, with Commision Internationale de l'Éclairage coordinate of $(0.21,0.47)$, at $10 \mathrm{~cd} \mathrm{~m}^{-2}$.

Keywords: TADF emitters, indolocarbazole, triazine, dimer, blue emitters, orientation, outcoupling effect, solution-processed OLEDs. 


\section{Introduction}

Organic thermally activated delayed fluorescence (TADF) materials have elicited tremendous excitement as an alternative to phosphorescent complexes as organic lightemitting diodes (OLEDs) because these organic compounds can also achieve a theoretical $100 \%$ internal quantum efficiency (IQE) but do not require the use of scarce, noble metals. ${ }^{1}$ Since the luminescence in an OLED is achieved through the radiative decay of electrically generated excitons, high-efficiency devices must be able to harvest both the $25 \%$ singlet and $75 \%$ triplet excitons to produce light. ${ }^{2}$ Distinct from phosphorescent compounds, TADF molecules harvest triplet excitons by converting them into emissive singlets via a reverse intersystem crossing (RISC) mechanism. This mechanism is operational when the energy gap $\left(\Delta E_{\mathrm{ST}}\right)$ between the lowest-lying singlet and triplet excited states $\left(\mathrm{S}_{1}\right.$ and $\left.\mathrm{T}_{1}\right)$ is sufficiently small and spin-orbit coupling (SOC) is nonnegligible. ${ }^{3-6}$ This small $\Delta E_{\text {ST }}$ can be achieved by spatially separating the highest occupied molecular orbital (HOMO) and the lowest unoccupied molecular orbital (LUMO), thereby reducing the exchange integral of these two orbitals determining the energies of the $S_{1}$ and $\mathrm{T}_{1}$ states relative to the ground state. Also note that due to their spatial separation, these states and their transitions to the ground state have predominantly charge transfer (CT) character. Highly twisted donor-acceptor architectures are typically employed to realize small $\Delta E_{\mathrm{ST} .}{ }^{3,7} \mathrm{SOC}$ can be enhanced by ensuring that the nature of the $\mathrm{S}_{1}$ and $\mathrm{T}_{1}$ states is different, for example by additionally involving a third (local) triplet state with different symmetry, because otherwise SOC vanishes when the orbital types for these two states are the same, according to El-Sayed's rule. ${ }^{8}$

Indolocarbazole (ICz) based emitters have been recently employed in several highperformance and highly horizontally oriented materials. ICz acts as a weak, planar, and rigid donor.9,10 Examples of compounds incorporating an $\mathrm{ICz}$ unit include reports from Xiang et al. with the emitters IndCzpTr1 and IndCzpTr2,11 and Maeng et al. with the emitter TRZ-TPDICz (Figure 1). ${ }^{12}$ In the doped film, IndCzpTr1 and IndCzpTr2 present high photoluminescence quantum yields, $\Phi_{\mathrm{PL}}$, of $75.2 \%$ and $71.9 \%$, respectively, and delayed fluorescence lifetimes, $\tau_{\mathrm{d}}$, of $25.48 \mu \mathrm{s}$ and $34.31 \mu \mathrm{s}$, respectively. The devices produced with these materials reached maximum external quantum efficiencies (EQE $\left.E_{\max }\right)$ values of $14.5 \%$ and $30 \%$ at low brightness, but efficiency roll-off was significant, with 
EQE at $100 \mathrm{~cd} \mathrm{~m}^{-2}, \mathrm{EQE}_{100}$, of $11.0 \%$ and $15.3 \%$ for the OLEDs with IndCzpTr1 and IndCzpTr2, respectively. The addition of two phenyl units on the ICz in TRZ-TPDICz increased the donor strength and led to $\Phi_{\mathrm{PL}}$ of near unity (96\%) and a much shorter $\tau_{\mathrm{d}}$ of $8.57 \mu \mathrm{s}$ in $20 \mathrm{wt} \%$ DBFPO film (DBFPO $=2$ 2,8-bis(diphenylphosphine oxide) dibenzofuran). The device made from this material has a very high $\mathrm{EQE}_{\max }$ of $30.3 \%$, which decreases to $18.4 \%$ at $1000 \mathrm{~cd} \mathrm{~m}^{-2}$; the use of a stronger donor in TRZ-TPDICz results in a red-shift of the electroluminescence, compared to IndCzpTr1 and IndCzpTr2 (the electroluminescence maximum wavelength, $\lambda_{\mathrm{EL}}$ of $472 \mathrm{~nm}$ and $496 \mathrm{~nm}$ for IndCzpTr1 and IndCzpTr2, respectively, against $\lambda_{\mathrm{EL}}$ of $509 \mathrm{~nm}$ for TRZ-TPDICz). In our previous work, we presented the first example of a di-functionalized ICz-based emitter ICzTRZ,13,14 that presented nearly complete horizontal orientation in a wide number of host matrices. The best combination of properties was obtained in mCBP as a host, with the photoluminescence maximum wavelength, $\lambda_{\mathrm{PL}}$ of $479 \mathrm{~nm}, \Phi_{\mathrm{PL}}$ of $70 \%$, and a $\tau_{\mathrm{d}}$ of 121.1 $\mu \mathrm{s}$. The anisotropy factor $(a)$ in $5 \mathrm{wt} \% \mathrm{mCBP}$ film is 0.09 , indicating a very high degree of horizontal orientation (91\%), which together with the high quantum yield led to a highperforming device with $\mathrm{EQE}_{\max }$ of $22.1 \%$.

It has been documented in the literature that some multichromophore emitters show enhanced molar extinction coefficients of absorption and high $\Phi_{\mathrm{PL}} \cdot{ }^{15-20}$ This led to OLEDs employing dual or multi emitter-designed compounds to show much improved $\mathrm{EQE}_{\max }$ compared to devices with their single-emitter counterparts (Figure 1), albeit with a redshifted emission. ${ }^{18-21}$ The advantages of the dual-emitter design are best illustrated by the cross-comparison of CzTRZ, ${ }^{22,23}$ a molecule that did not present any TADF and thus the OLED showed a low EQE max $_{\text {of }} 5.8 \%$, while the emitter, 33TCzTTrz, ${ }^{24}$ is TADF and the OLED showed a much superior EQE $E_{\max }$ of $25.0 \%$. There is a significant red-shift of the electroluminescence, with $\lambda_{\mathrm{EL}}$ going from $449 \mathrm{~nm}$ for CzTRZ to $490 \mathrm{~nm}$ for 33TCzTTrz. 

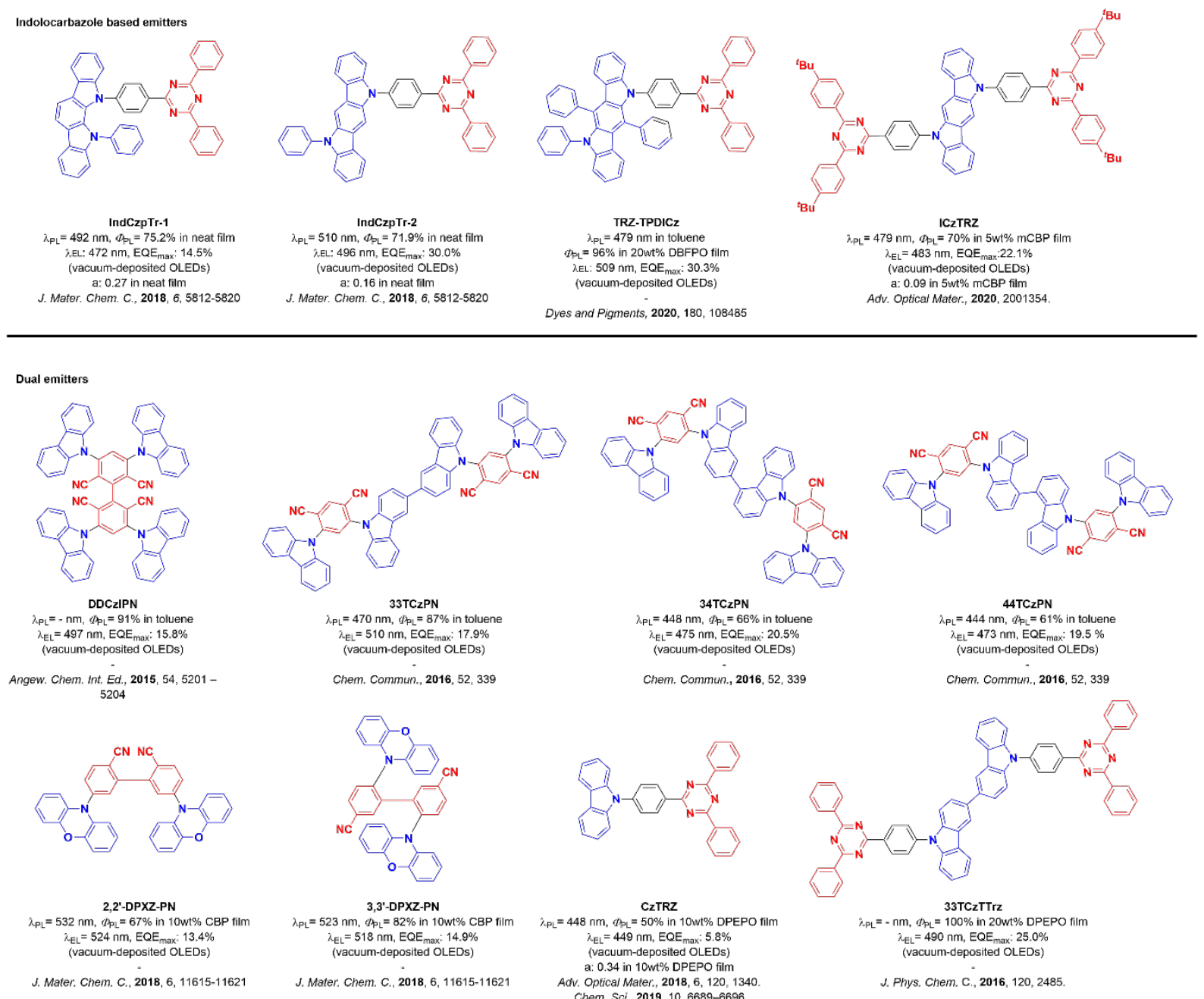

Chem. Commun., 2016, 52, 339

Chem. Commun., 2016, 52, 339

Chem. Commun., 2016, 52, 339
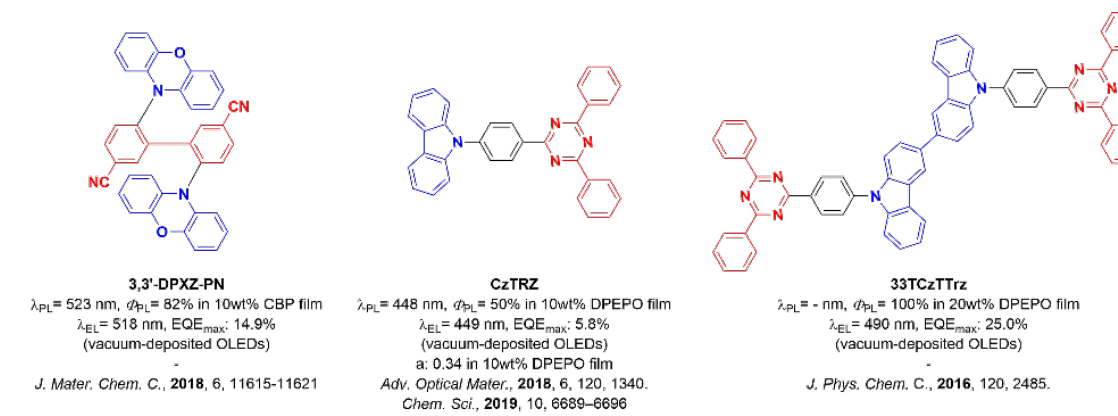

Figure 1. Molecular structures of emitters.

In this work, we utilized a similar strategy to assess the change in optoelectronic properties and device performance of the compound DICzTRZ compared to our recently reported ICzTRZ study. ${ }^{14}$ We note that the effective doubling of the molecular weight necessitates that we fabricate solution-processed devices. Importantly, solutionprocessed films tend to present isotropic orientation ${ }^{25}$ due to the slower deposition times coupled with higher degree of freedom of movement in the solution, unlike the orientation of the emitter in vacuum-deposited films, which occurs only at the surface of the film where the emitter orientation is then "frozen" into place once additional layers of material have covered it. While this loss of controlled orientation in the solution-processed film is true for small molecules, polymers and other high molecular weight emitters can show at least some degree of orientation in solution-processed films. For instance, Senes et al.26,27 showed that the OPVn series of polymers exhibited higher horizontal orientation by 
increasing the length of the polymer chain, and by extension the molecule. Considering the high degree of horizontal orientation that ICzTRZ already showed in vacuumdeposited films (anisotropy factor of 0.09 in $10 \mathrm{wt} \%$ film of mCBP) and the high molecular weight of DICzTRZ, we hypothesized that DICzTRZ may also present horizontal orientation in the film and subsequently improve light outcoupling in the device.

a)

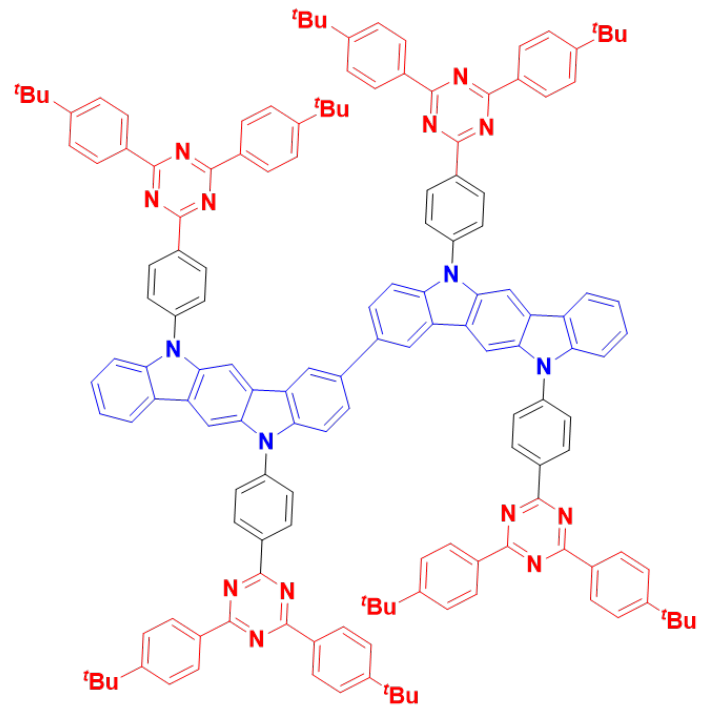

b)

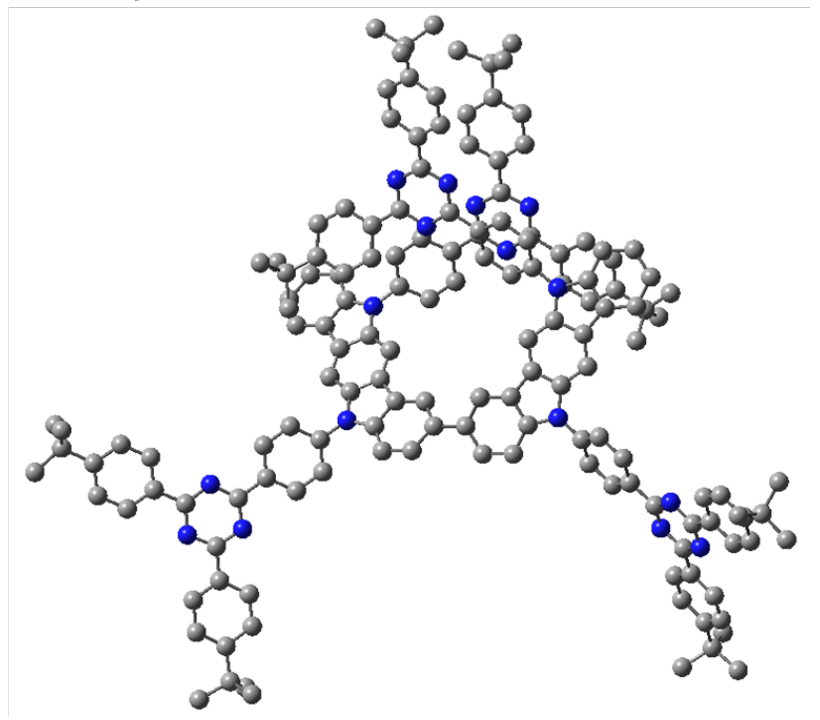

Figure 2. a) Molecular structure and b) optimized DFT-calculated geometry of DICzTRZ.

\section{Synthesis}

The oxidative coupling conditions for the synthesis of carbazole dimers were initially applied to access the dimer of ICzTRZ. ${ }^{28,29}$ Treating ICzTRZ with $\mathrm{FeCl}_{3}$ in DCM at room temperature for 12 hours did not lead to any product formation. However, when the temperature was increased to $40{ }^{\circ} \mathrm{C}$, DICzTRZ was formed and was isolated in a yield of $20 \%$, while increasing the temperature to $60^{\circ} \mathrm{C}$ resulted in complete consumption of the starting material and DICzTRZ was isolated in 66\% yield. The identity and purity of DICzTRZ were determined by a combination of NMR spectroscopy, mass spectrometry, and IR spectroscopy.

\section{Theoretical calculations}



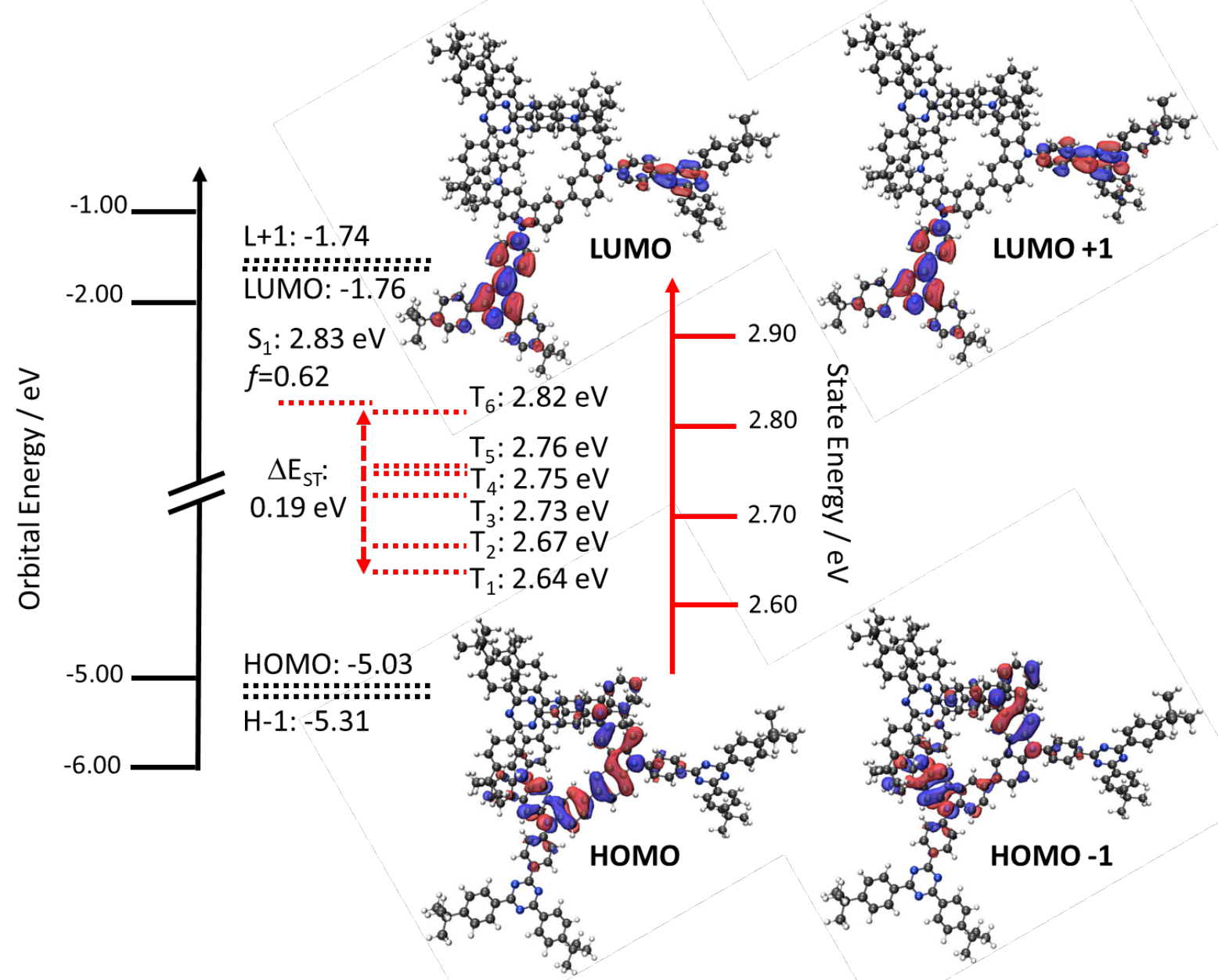

Figure 3. HOMO, HOMO-1 (H-1), LUMO, and LUMO+1 (L+1) electron density distributions (isovalue: 0.02) and energy levels, excited state energy levels.

Density functional theory (DFT) and time-dependent DFT (TD-DFT) calculations in the gas phase at the PBE0/6-31G(d,p) level reveal the potential of DICzTRZ as a TADF material (Figure 3 ). The nature of the $S_{1}$ and $T_{1}$ states and their corresponding energies were then obtained using the Tamm-Dancoff approximation ${ }^{30}$ to TD-DFT (TDA-DFT). DICzTRZ possesses a $\Delta E_{\text {ST }}$ of $0.19 \mathrm{eV}$, comparable to $0.22 \mathrm{eV}$ obtained for ICzTRZ at the same level of theory. We can observe a slightly stabilized $S_{1}$ energy of $2.83 \mathrm{eV}(2.92 \mathrm{eV}$ for ICzTRZ) and $\mathrm{T}_{1}$ energy of $2.64 \mathrm{eV}\left(2.70 \mathrm{eV}\right.$ for ICzTRZ ${ }^{14}$ compared to those of ICzTRZ. Compared to ICzTRZ, there is a much higher density of intermediate triplet states between $S_{1}$ and $T_{1}$, which is expected to enhance the efficiency of the RISC process due to 
the presence of increased spin-vibronic coupling. ${ }^{31-36}$ The permanent dipole moment (PDM) of DICzTRZ is substantial increased to 2.1 Debye compared to that in ICzTRZ (0.3 Debye); however, both the transition dipole moment (TDM) and oscillator strength ( $f$ ) are slightly smaller (TDM $=7.6$ Debye and $f=0.62$ ) than the values calculated for ICzTRZ (TDM = 7.9 Debye and $f=0.72$ ). DICzTRZ shows a shallower HOMO at $-5.03 \mathrm{eV}$, reflective of a certain degree of conjugation between the two indolocarbazole moieties, compared to the HOMO of ICzTRZ $(-5.19 \mathrm{eV})$. The LUMO level remains essentially unchanged $(-1.76$ eV for DICzTRZ vs -1.75 for ICzTRZ) since the electronic environment surrounding the ${ }^{t} \mathrm{Bu}$-triazine remains essentially unperturbed.

\section{Optoelectronic properties}

The electrochemical properties of the two materials were studied in degassed DCM with tetra- $n$-butylammonium hexafluorophosphate as the electrolyte and $\mathrm{Fc} / \mathrm{Fc}^{+}$as the internal reference (Figure 4a), data are reported versus a Saturated Calomel Electrode (SCE). In both DICzTRZ and ICzTRZ ${ }^{14}$ we observed a reversible oxidation wave with respective oxidation potential $\left(E_{\mathrm{ox}}\right)$ at $0.87 \mathrm{~V}$ and $0.96 \mathrm{~V}$ vs SCE. Both compounds also present a second oxidation wave that is more prominent and cathodically shifted for DICzTRZ at $1.05 \mathrm{~V}$, compared to $1.14 \mathrm{~V}$ for ICzTRZ. No reduction wave is observed for DICzTRZ. The HOMO value calculated from the oxidation potential obtained from differential pulse voltammetry (DPV), is $-5.21 \mathrm{eV}$, which is stabilized compared to that predicted from DFT ( $E_{\text {номо }}-5.03 \mathrm{eV}$ ); however, the less positive oxidation potentials in DICzTRZ versus ICzTRZ does align with the predictions obtained by DFT.

The UV-vis absorption spectrum of DICzTRZ, while slightly red-shifted and with higher molar absorptivity (as was the case for previously published multichromophore materials), ${ }^{15-20}$ coincides closely with the one from ICzTRZ ${ }^{14}$ (Figure $\mathbf{4 b}$ ) and also with other indolocarbazole-based compounds. ${ }^{11}$ The nearly identical profile leads us to conclude that the character of the transitions are likely to be very similar to those associated with ICzTRZ, showing CT character for the two absorption bands between 330 and $350 \mathrm{~nm}$, between the diindolocarbazole donor and the two triazine acceptors, while the second lower energy band one presents LE (on the indolocarbazole) character. The two lower energy and lower absorptivity bands at $390 \mathrm{~nm}$ and $410 \mathrm{~nm}$ are both assigned to CT-type transitions. 
Solvatochromic studies (Figure 4c) for DICzTRZ show that the PDM of the ground state structure is small and so the absorption spectrum is essentially not affected by changes in polarity, while the excited state shows the characteristic positive solvatochromism associated with an emission from a CT state $\left(\lambda_{\mathrm{PL}}\right.$ going from $462 \mathrm{~nm}$ in the least polar methyl-cyclohexane to $548 \mathrm{~nm}$ in the most polar dichloromethane). From the previously calculated HOMO level determined from DPV and the optical gap obtained from the intersection of the normalized absorption and emission spectra in DCM $\left(E_{\text {gap }}=2.71 \mathrm{eV}\right)$, we were able to obtain a LUMO value of $-2.50 \mathrm{eV}$.

a)
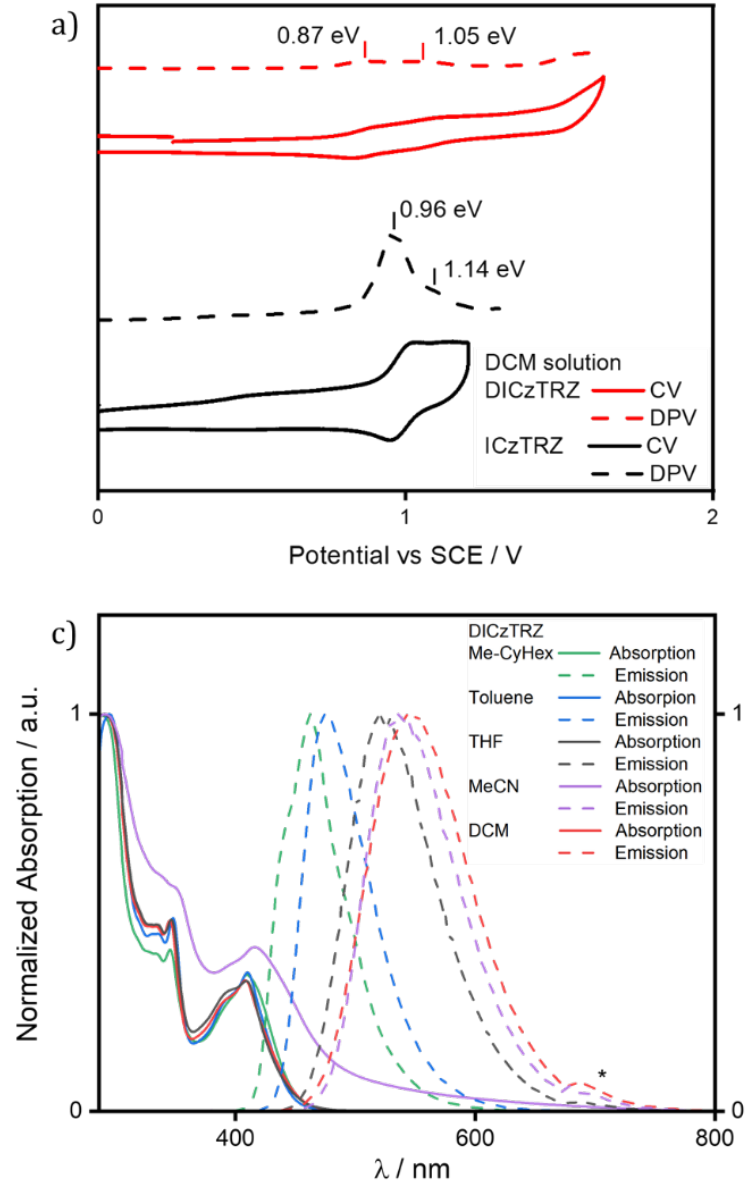

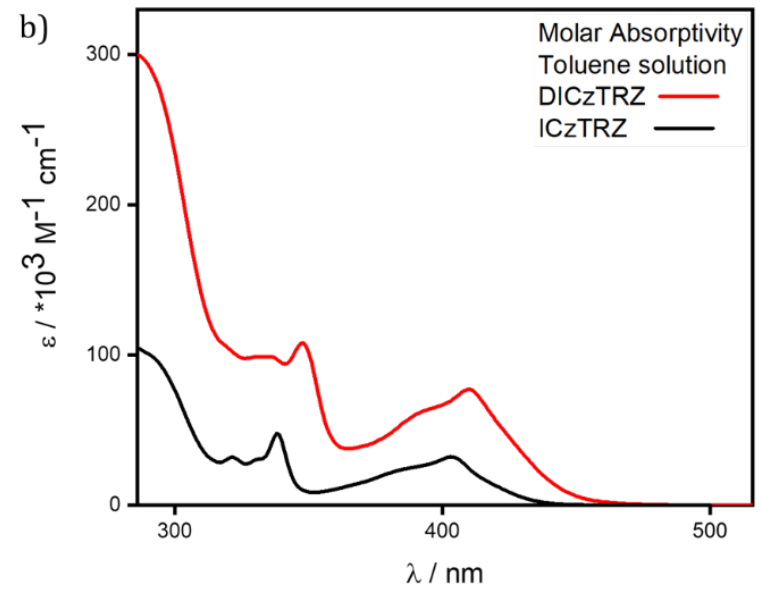

d)

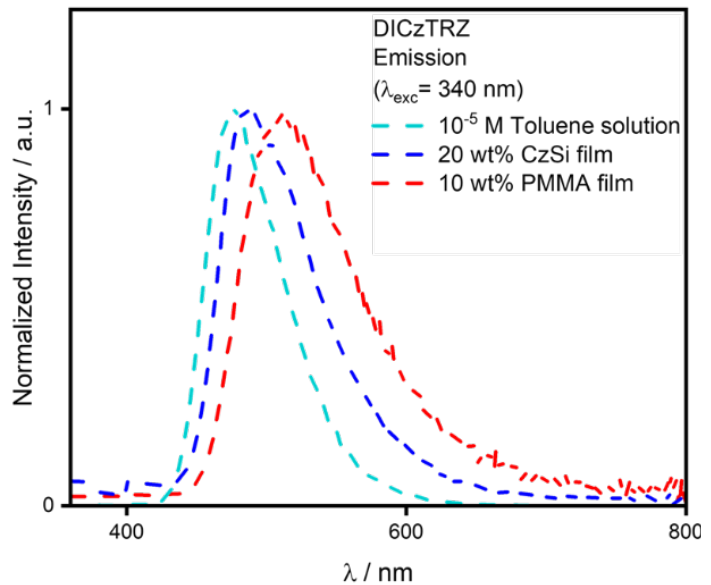

Figure 4. a) Cyclic voltammetry (CV) and differential pulse voltammetry (DPV) of DICzTRZ in DCM (scan rate $=100 \mathrm{mV} / \mathrm{s}$ ). b) UV-vis absorption spectrum of DICzTRZ in $10^{-5} \mathrm{M}$ toluene solution. c) Ground and excited state solvatochromism study of DICzTRZ (excitation wavelength, $\lambda_{\mathrm{exc}},=340 \mathrm{~nm},{ }^{*}=$ second harmonic of the excitation source); $\mathrm{d}$ ) emission spectra of DICzTRZ in $10^{-5} \mathrm{M}$ toluene solution (cyan), $20 \mathrm{wt} \% \mathrm{CzSi}$ film (blue), and $10 \mathrm{wt} \%$ PMMA film (red), $\left(\lambda_{\mathrm{exc}}=340 \mathrm{~nm}\right)$. 
The emission of DICzTRZ in degassed toluene is red-shifted at $477 \mathrm{~nm}$ compared to ICzTRZ, ${ }^{14}$ at $462 \mathrm{~nm}$ (Figure 4d). The excitation spectrum mirrors the profile of the UVvis absorption (Figure S4a). Transient PL measurements in degassed toluene show monoexponential prompt and delayed fluorescence decays at $8.94 \mathrm{~ns}$ and $28.83 \mu \mathrm{s}$, respectively (Figure S4c-d). After exposure to oxygen, the delayed fluorescence disappears while the prompt decay lifetime, $\tau_{\mathrm{p}}$, is slightly reduced to $6.80 \mathrm{~ns}$, implying the involvement of triplet states in the emission. When compared to ICzTRZ in degassed toluene, DICzTRZ presents comparable $\tau_{\mathrm{p}}$ (9.0 ns for ICzTRZ), while we observe a substantial one order of magnitude decrease in the delayed lifetime, $\tau_{\mathrm{d}},\left(229.2 \mu\right.$ s for ICzTRZ $\left.^{14}\right)$, reflective of a more efficient RISC process. DICzTRZ is less emissive than ICzTRZ ( $\Phi_{\mathrm{PL}}$ of $72 \%{ }^{14}$ ), with $\Phi_{\mathrm{PL}}$ in degassed toluene of $60 \%$ that decreases to $44 \%$ once exposed to oxygen. This reduction in $\Phi_{\mathrm{PL}}$ is in part due to the decrease in the radiative decay rate given the smaller calculated oscillator strength for the emissive $S_{1}$ state for this compound compared to ICzTRZ. The $\Delta E_{S T}$ of DICzTRZ in toluene glass at $77 \mathrm{~K}$ is $0.21 \mathrm{eV}$ (see Figure S4, which is significantly smaller than the $0.32 \mathrm{eV}$ obtained for ICzTRZ under the same conditions. The $\mathrm{T}_{1}$ levels of both DICzTRZ and ICzTRZ are comparable at $2.59 \mathrm{eV}$ and $2.62 \mathrm{eV}$, respectively, while the $S_{1}$ level for DICzTRZ is more stabilized at $2.80 \mathrm{eV}$ vs $2.94 \mathrm{eV}$ for ICzTRZ). We can clearly observe that the phosphorescence spectrum presents a welldefined structure, typical for transitions coming from a local excited (LE) type state on the di-indolocarbazole. TDA-DFT calculations in the gas phase predict that the $\mathrm{T}_{1}$ state is CT in nature while the lowest-lying triplet states with LE character $\operatorname{are} \mathrm{T}_{3}$ and $\mathrm{T}_{4}\left(\mathrm{~T}_{3}\right.$ and $\mathrm{T}_{4}$ are at $2.73 \mathrm{eV}$ and $2.75 \mathrm{eV}$, respectively, while $\mathrm{T}_{1}$ is at $2.64 \mathrm{eV}$, See Table $\mathbf{S} \mathbf{1}$ and Figure S3. The character of the different transitions was also evaluated by analysis of the Natural Transition Orbitals (NTOs) (See Table S2). The $\mathrm{T}_{1}$ and $\mathrm{T}_{2}$ HONTO and LUNTO (Highest Occupied and Lowest Unoccupied Natural Transition Orbitals) are localized on the central di-indolocarbazole and adjacent triazine, respectively, showing a clear CT between donor and acceptor moieties in the molecule. As previously mentioned, $\mathrm{T}_{3}$ and $\mathrm{T}_{4}$ present $\mathrm{LE}$ character, with the NTOs localized mainly the central di-indolocarbazole. The character of each of $\mathrm{T}_{5}$ and $\mathrm{T}_{6}$ is more difficult to assign as the electron density of the transition is localized on one of the indolocarbazole-triazine fragments and showing a high degree of overlap between the HONTO and LUNTO, which indicates a transition with a mixed CT 
and LE character. $S_{1}$ also presents a clear CT transition from the di-indolocarbazole to the triazine.
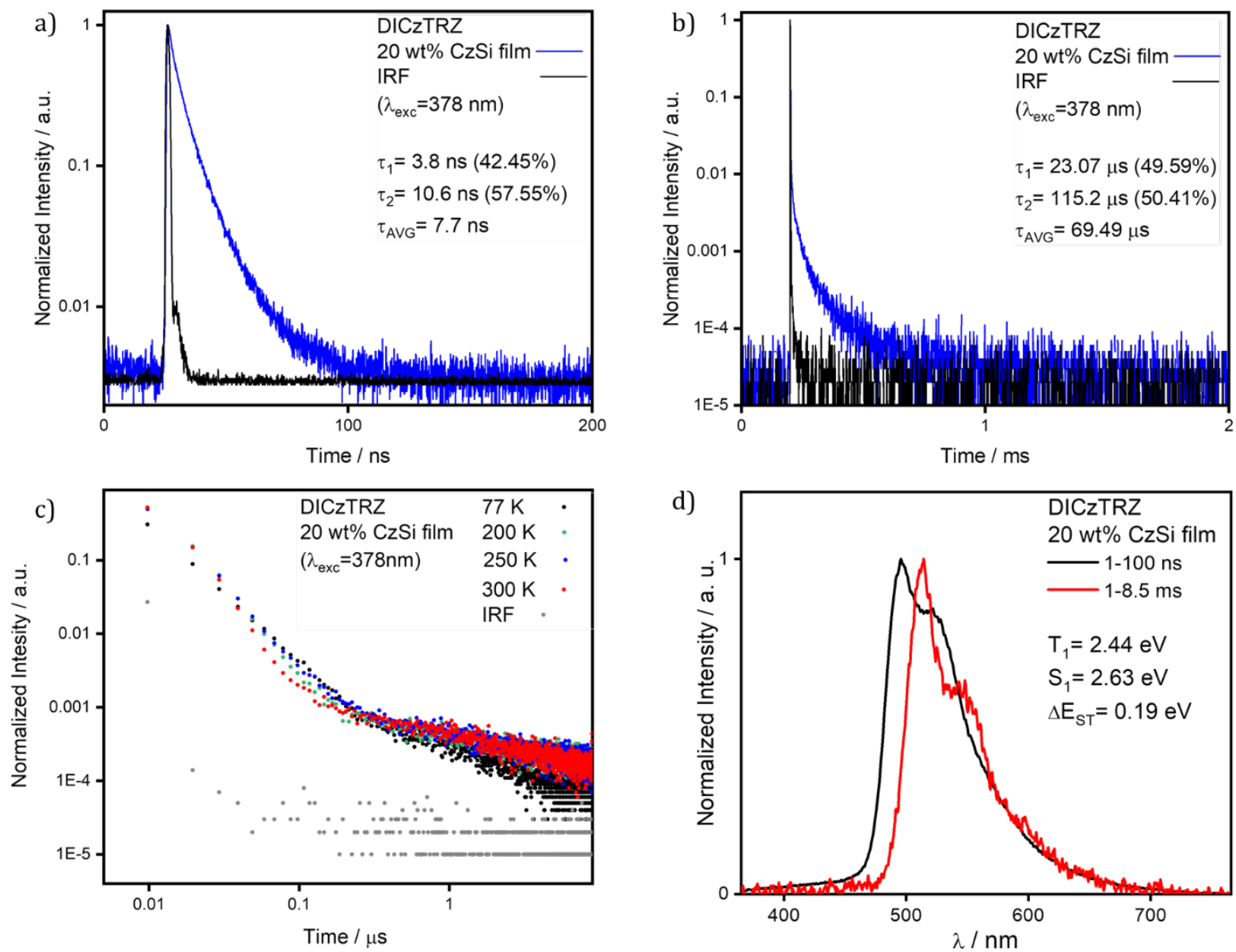

Figure 5. a) Prompt and b) Delayed time-resolved decay in spin-coated $20 \mathrm{wt} \% \mathrm{CzSi}$ film of DICzTRZ $\left.\left(\lambda_{\text {exc }}=378 \mathrm{~nm}\right) ; \mathrm{c}\right)$ Delayed fluorescence decay data measured at different temperatures in spin-coated 20 wt $\%$ CzSi film of DICzTRZ $\left(\lambda_{\text {exc }}=378 \mathrm{~nm}\right)$; d) Prompt fluorescence and phosphorescence spectra at $77 \mathrm{~K}$ in drop-casted $20 \mathrm{wt} \% \mathrm{CzSi}$ film $\left(\lambda_{\text {exc }}\right.$ $=343 \mathrm{~nm}$, prompt and delayed fluorescence spectra were obtained in the 1-100 ns and 1-10 ms time range, respectively).

With a view to incorporating DICzTRZ as the emitter in a solution-processed OLED, we next investigated the photophysical behavior of this compound in solid host matrices. We began with 10 wt\% doped film of DICzTRZ in PMMA as the polarity of PMMA emulates well that of toluene. ${ }^{37}$ The emission maximum in PMMA is $514 \mathrm{~nm}$ with a corresponding $\Phi_{\mathrm{PL}}$ of $29 \%$ under $\mathrm{N}_{2}$. The significantly red-shifted emission in the PMMA film compared to that in toluene coupled with a significantly lower $\Phi_{\mathrm{PL}}$ is suggestive that aggregation- 
caused quenching is prevalent in this host matrix. Transient PL measurement (Figure S5 showed multiexponential decay kinetics and lifetimes with an average $\tau_{\mathrm{p}}$ of $8.6 \mathrm{~ns}$ [ $\tau_{1}=3.5$ ns (37.5\%), $\left.\tau_{2}=11.6 \mathrm{~ns}(62.5 \%)\right]$ and an average $\tau_{\mathrm{d}}$ of $156.1 \mu \mathrm{s}\left[\tau_{1}=27.98 \mu \mathrm{s}(39.5 \%)\right.$, $\left.\tau_{2}=239.7 \mu \mathrm{s}(60.5 \%)\right]$, respectively. The average prompt fluorescence lifetimes are of a similar magnitude to that of ICzTRZ $\left(\tau_{\mathrm{p}}=11.5 \mathrm{~ns}\right)$ while the average delayed fluorescence decays much faster for DICzTRZ ( $\tau_{\mathrm{d}}=252.8 \mu$ s for ICzTRZ). We next focused on the photophysical study in a suitably high triplet energy small molecule host material, $\mathrm{CzSi}$ (9-(4-tert-butylphenyl)-3,6-bis(triphenylsilyl)-9H-carbazole). The emission in $\mathrm{CzSi}$ at $488 \mathrm{~nm}$, is only slightly red-shifted compared to that in toluene. Gratifyingly, the $\Phi_{\mathrm{PL}}$ is substantially higher at 57\% in 20 wt\% doped CzSi film, compared to the $10 \mathrm{wt} \%$ PMMA films (Table 1). In this host, transient PL measurements show the presence of both prompt and delayed fluorescence with respective average lifetimes of $\tau_{\mathrm{p}} 7.7 \mathrm{~ns}$ [ $\tau_{1}=3.8 \mathrm{~ns}(42.5 \%)$, $\left.\tau_{2}=10.6 \mathrm{~ns}(57.6 \%)\right]$ and $\tau_{\mathrm{d}}$ of $69.49 \mu \mathrm{s}\left[\tau_{1}=23.07 \mu \mathrm{s}(49.6 \%), \tau_{2}=115.2 \mu \mathrm{s}(50.4 \%)\right]$. While the $\Phi_{\mathrm{PL}}$ largely benefits from the change in the host, the lifetimes of the prompt fluorescence remain largely unchanged while we observe a much shorter delayed fluorescence. Both prompt and delayed lifetimes of DICzTRZ in CzSi are shorter than those of ICzTRZ in the same host ( $\tau_{\mathrm{p}} 9.5 \mathrm{~ns}, \tau_{\mathrm{d}}$ of $147.3 \mu \mathrm{s}$ ). The $\Delta E_{\mathrm{ST}}$ values in CzSi (Figure 5d) and PMMA (Figure S5), are $0.19 \mathrm{eV}$ and 0.03, respectively. From a cross-comparison of the state energies (Table 1 ) we can see that the $\mathrm{T}_{1}$ state remains essentially the same regardless of the environment, this due to the LE nature of this excited state. The energy of the $S_{1}$ state varies with the environments (with energies of $2.94 \mathrm{eV}, 2.72 \mathrm{eV}$, and 2.75 $\mathrm{eV}$ for toluene solution, CzSi film and PMMA film, respectively for ICzTRZ), characteristic of a CT type state, but the shape of the spectra in all media adopt a structured profile, typical for LE-type states, suggesting a state of mixed CT and LE character (Figure S4d and S5d). DICzTRZ and ICzTRZ possess comparable $\Delta E_{\mathrm{ST}}$ in $\mathrm{CzSi}$, at $0.19 \mathrm{eV}$ and $0.16 \mathrm{eV}$ respectively. Temperature-dependent time-resolved PL decays (Figure 5c) reveal the clear increase in the intensity of the delayed emission with higher temperature, a hallmark of TADF. 
Table 1. Photophysical properties of ICzTRZ ${ }^{14}$ and DICzTRZ.

\begin{tabular}{|c|c|c|c|c|c|c|c|}
\hline Material & Environment & $\lambda_{\mathrm{PL}} / \mathbf{n m}^{c}$ & $\Phi_{\mathrm{PL}} \mathrm{N}_{2}\left(\right.$ air) ${ }^{d} / \%$ & $\tau_{\mathbf{p}}, \tau_{\mathbf{d}} g / \mathbf{n s} ; \mu \mathbf{S}$ & $\begin{array}{l}S_{1}{ }^{h} \\
e V\end{array}$ & $\begin{array}{ll}\mathrm{T}_{1}{ }^{i} & / \\
\mathrm{eV} & \end{array}$ & $\begin{array}{l}\Delta E_{s \mathrm{~T}^{i}} / \\
\mathrm{eV}\end{array}$ \\
\hline \multirow[t]{3}{*}{ ICzTRZ $^{a}$} & Toluene $\left(10^{-5} \mathrm{M}\right)$ & 462 & $72(56)^{e}$ & $9.0 ; 229.2$ & 2.94 & 2.62 & 0.32 \\
\hline & $\mathrm{CzSi} 20 \mathrm{wt} \%$ & 475 & $63(50)^{f}$ & $9.5 ; 147.3$ & 2.72 & 2.56 & 0.16 \\
\hline & PMMA $10 \mathrm{wt} \%$ & 470 & $31(28)^{f}$ & $115 ; 252.8$ & 2.75 & 2.64 & 0.11 \\
\hline \multirow[t]{3}{*}{ DICzTRZ $^{b}$} & Toluene $\left(10^{-5} \mathrm{M}\right)$ & 477 & $60(44)^{e}$ & $8.9 ; 28.83$ & 2.80 & 2.59 & 0.21 \\
\hline & CzSi 20 wt $\%$ & 488 & $57(42)^{f}$ & $7.7 ; 69.49$ & 2.63 & 2.44 & 0.19 \\
\hline & PMMA 10 wt $\%$ & 514 & $29(22)^{f}$ & $8.6 ; 156.1$ & 2.61 & 2.58 & 0.03 \\
\hline
\end{tabular}

${ }^{a}$ previous work $14 ;{ }^{b}$ this work; ${ }^{c}$ measured at room temperature; $d \lambda_{\text {exc }}=340 \mathrm{~nm}$; ${ }^{e}$ obtained via the optically dilute method $^{38}$ (see SI), quinine sulfate (0.5 M) in $\mathrm{H}_{2} \mathrm{SO}_{4}$ (aq) was used as the reference, $\Phi_{\mathrm{PL}}: 54.6 \%, \lambda_{\text {exc }}=360 \mathrm{~nm} ; 39 f_{\text {obtained }}$ via integrating sphere; $g \tau_{\mathrm{p}}$ (prompt lifetime) and $\tau_{\mathrm{d}}$ (delayed lifetime) were obtained from the transient PL decay of degassed solution/doped film, $\lambda_{\mathrm{exc}}=378 \mathrm{~nm} ;{ }^{h} \mathrm{~S}_{1}$ was obtained from the onset of the prompt emission measured at 77 $\mathrm{K} ;{ }^{i} \mathrm{~T}_{1}$ was obtained from the onset of the phosphorescence spectrum measured at $77 \mathrm{~K} ; j \Delta E_{\mathrm{ST}}=\mathrm{S}_{1}-\mathrm{T}_{1}$.

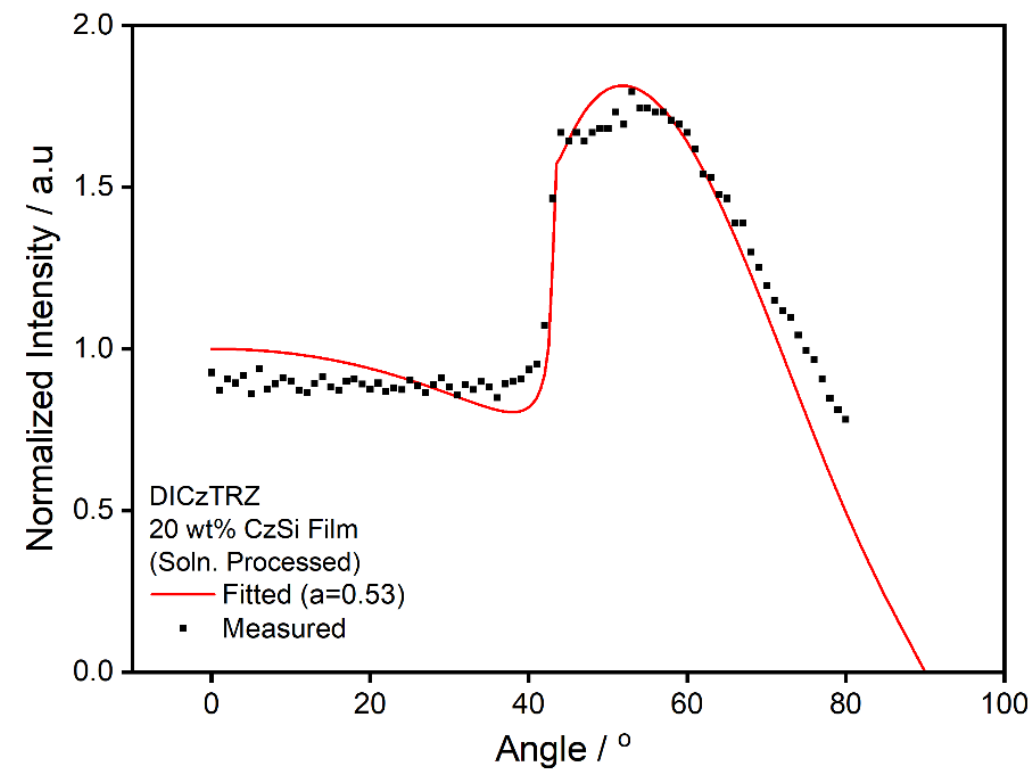

Figure 6. Angle-resolved photoluminescence measurement of a solution-processed film of 20 wt\% DICzTRZ in CzSi. The red line shows a fit using the dipole emission model as described in detail in the SI, yielding an anisotropy factor, $a$, of 0.53 (data taken at $\lambda_{\mathrm{em}}=$ $500 \mathrm{~nm})$.

Designing a molecule able to achieve RISC and the desired 100\% IQE is just the first step toward an efficient TADF material since the light needs to escape the OLED device. A device is composed of a stack of several layers of different materials (thus with different refractive indexes) between two electrodes that can lead to total internal reflection at the organic-glass as well as the glass-air interfaces and coupling to surface plasmon polaritons (SPP) at the interface with the cathode, lowering the EQE of the device to a 
maximum of about 25\%. An emitter will emit light perpendicularly to its TDM, which means that creating a structure able to achieve horizontal orientation of the TDMs, quantified by the anisotropy factor, $a$, in the film - where 1- $a$ is the fraction of horizontally aligned TDMs, will maximize the amount of light exiting the device.

Polarization and angle dependent luminescence spectroscopy was used to measure $a$ for solution processed films of 20 wt\% DICzTRZ in CzSi (9-(4-tert-butylphenyl)-3,6bis(triphenylsilyl)-9H-carbazole). The data were then analysed via optical simulation to yield and anisotropy factor of 0.53 , which disappointingly implies that the emitter presents a strongly vertical orientation (Figure 6); the optical simulation of $10 \mathrm{wt} \%$ DICzTRZ in mCP film is shown in Figure $\mathbf{S 6 .}$

\section{OLED devices}

Finally, DICzTRZ-based OLEDs were fabricated using the following device structure: ITO (indium tin oxide) (50 nm)/PEDOT:PSS (poly(3,4-ethylenedioxythiophene) polystyrene sulfonate) (35 nm)/PVK (poly(9-vinylcarbazole)) $(10 \mathrm{~nm}) / X$ wt\% DICzTRZ: CzSi (20 $\mathrm{nm}$ )/PPF (2,8-bis(diphenylphosphoryl)dibenzo[b,d]furan) (5 nm)/TPBi (1,3,5-tris(1phenyl-1H-benzo[d]imidazol-2-yl)benzene) (50 nm)/Liq (lithium quinolin-8-olate) (1 $\mathrm{nm}) / \mathrm{Al}(80 \mathrm{~nm})$, where $X$ is 20 or 30 . The PVK layer is applied to facilitate hole injection from PEDOT:PSS to the emitting layer. Besides, PVK and PPF, possessing high $\mathrm{T}_{1}$ energies of $3.0 \mathrm{eV}^{40}$ and $3.1 \mathrm{eV},{ }^{41}$ respectively, were inserted to confine the excitons in the emitting layer. PEDOT:PSS, PVK and the emitting layer were fabricated by spin-coating, and the other layers were vacuum-deposited. Device characteristics are shown in Figure $\mathbf{7}$ and the device performance is summarized in Table 2. As shown in Table 2, $20 \mathrm{wt} \%$ DICzTRZbased OLEDs achieved EQE $E_{\max }$ of $8.0 \%$ and $\lambda_{\mathrm{EL}}$ of $492 \mathrm{~nm}$ with CIE coordinates $(x, y)$ of $(0.21,0.47)$ at $10 \mathrm{~cd} \mathrm{~m}^{-2}$. 

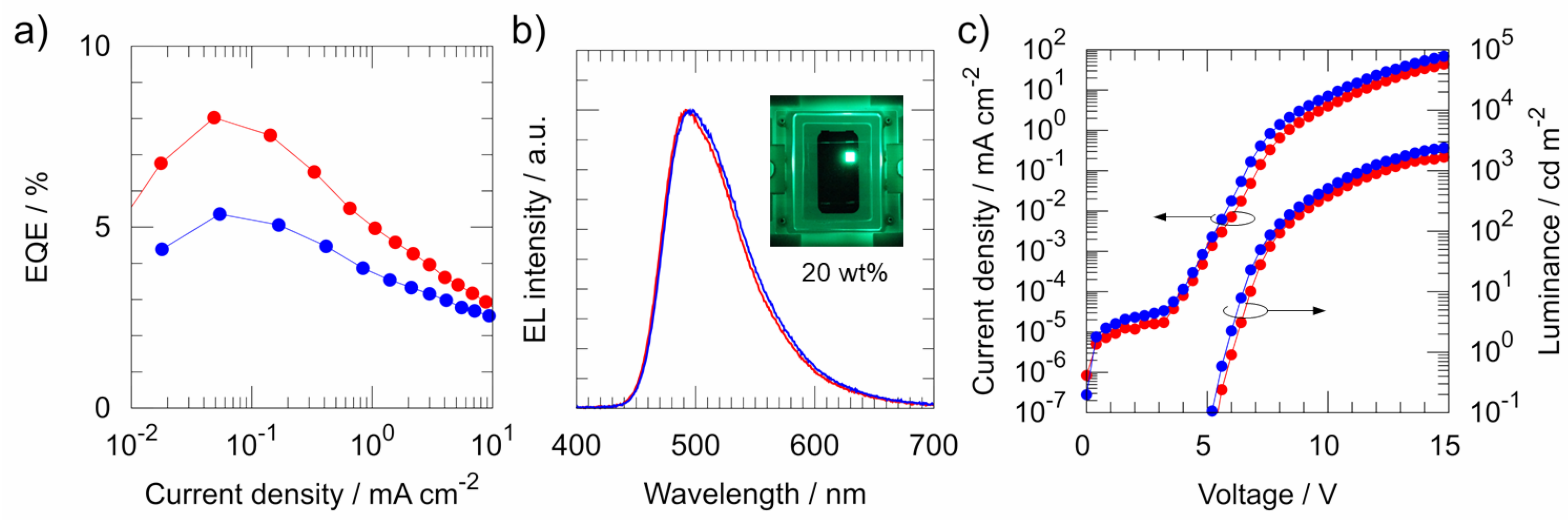

Figure 7. Device characteristics of 20 and 30 wt\% DICzTRZ-based OLEDs, which are represented by red and blue, respectively. a) EQE-current density b) EL spectra and c) current density-voltage-luminance properties.

Table 2. Device performances of $X$ wt $\%$ DICzTRZ-based OLEDs (where $X=20,30$ ).

\begin{tabular}{cccc}
\hline Concentration $/ \%$ & $\mathrm{EQE}_{\max } / \%$ & $\lambda_{\mathrm{EL}} / \mathrm{nm}^{\mathrm{a}}$ & $\mathrm{CIE}(x, y)$ \\
\hline 20 & 8.0 & 492 & $(0.21,0.47)$ \\
30 & 5.4 & 498 & $(0.22,0.49)$ \\
\hline
\end{tabular}

${ }^{a}$ Determined from EL spectrum at $1 \mathrm{~mA} \mathrm{~cm}^{-2}$.

We next simulated the device EQE (Figure S7). As shown in Figure 8, with the pre-determined parameters ( $\Phi_{\mathrm{PL}}$ and $a$ ) along with the optical constants of the different materials in the OLED stack, we predict the device to show an $\mathrm{EQE}_{\max }$ of $9 \%$, which aligns with the measured $\mathrm{EQE}_{\max }$. The simulation also demonstrates that at the given $\Phi_{\mathrm{PL}}$ of $57.1 \%$, a device EQE in the range of $10 \%$ is a result of the vertical orientation of the emitter within the EML. 


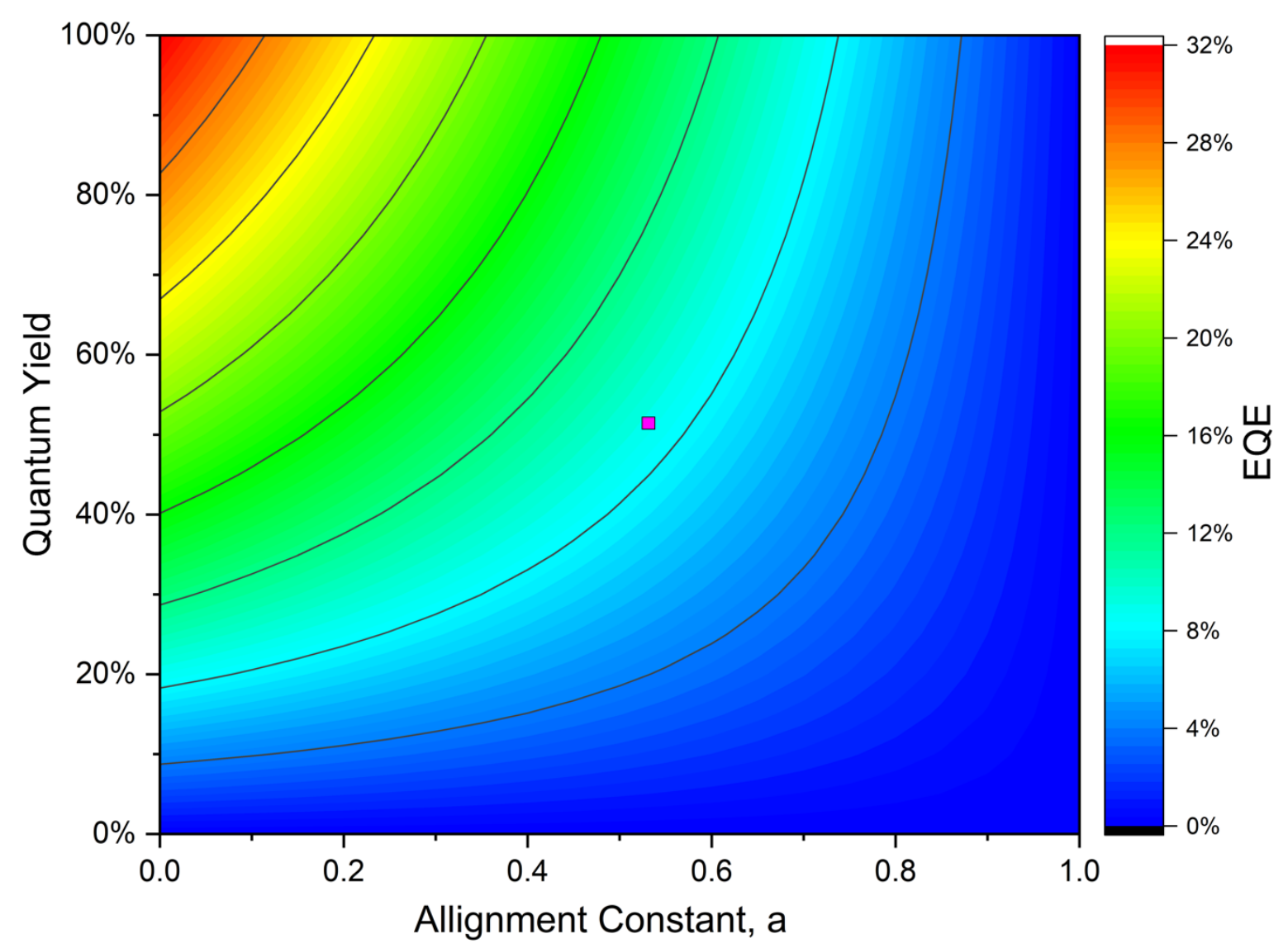

Figure 8. Device efficiency simulation of fabricated 20wt\% DICzTRZ-based OLEDs depicting the variation in EQE with varied PL Quantum Yield (vertical axis) and anisotropy factor (horizontal axis). Predicted EQE indicated with a pink rectangular mark which agrees with the experimental results.

\section{Conclusions}

Building upon our previously reported emitter, ICzTRZ, here we presented a dual emitter strategy consisting of two ICzTRZ moieties covalently linked together in the form of DICzTRZ. DFT calculations showed a much larger density of triplet states, which suggests that RISC will be faster in this compound compared to its parent. The twin design strategy leads to an enhancement in the molar extinction coefficient of the low-lying CT states, accompanied by a red-shift in the emission. The $20 \mathrm{wt} \%$ doped CzSi film of DICzTRZ emits in the blue at $488 \mathrm{~nm}$ and shows a photoluminescence quantum yield of $57.1 \%$. Unfortunately, the TDM of this material is not preferentially horizontally oriented in the solution-processed film. The OLED shows an EQE $\mathrm{E}_{\max }$ of $8.0 \%$ and $\lambda_{\mathrm{EL}}$ of $492 \mathrm{~nm}$. 


\section{Supporting information}

Synthesis protocols, NMR spectra, supplementary photophysical measurements, computational data obtained from DFT and TD DFT and electroluminescence data.

\section{Acknowledgments}

We (EC, BAN, PS, WB \& EZ-C) thank EU Horizon 2020 Grant Agreement No. 812872 (TADFlife) for funding. This project is supported by the Helmholtz Association Program at the Karlsruhe Institute of Technology (KIT). The German Research Foundation (formally Deutsche Forschungsgemeinschaft DFG) in the framework of SFB1176 Cooperative Research Centre "Molecular Structuring of Soft Matter" (CRC1176, A4, B3, C2, C6) and the cluster 3D Matter Made To Order all funded under Germany's Excellence Strategy -2082/1--390761711 are greatly acknowledged for financial contributions. We acknowledge support from the Engineering and Physical Sciences Research Council of the UK (grant EP/P010482/1), from the International Collaborative Research Program of Institute for Chemical Research, Kyoto University (grant \# 2020-37 and 2021-37), and from JSPS KAKENHI Grant Number JP20H05840 (Grant-in-Aid for Transformative Research Areas, "Dynamic Exciton”). ZZ acknowledges the financial support from Chinese Scholarship Council (CSC, 201606890009) for his PhD studies. 


\section{References}

(1) Liu, Y.; Li, C.; Ren, Z.; Yan, S.; Bryce, M. R. All-Organic Thermally Activated Delayed Fluorescence Materials for Organic Light-Emitting Diodes. Nat. Rev. Mater. 2018, 3, 18020 .

(2) Baldo, M. A.; an dY. You, D. F. O.; Shoustikov, A.; Sibley, S.; Thompson, M. E.; Forrest, S. R. Highly Efficient Phosphorescent Emission from Organic Electroluminescent Devices. Nature 1998, 395 (September), 151-154.

(3) Milián-Medina, B.; Gierschner, J. Computational Design of Low Singlet-Triplet Gap All-Organic Molecules for OLED Application. Org. Electron. 2012, 13 (6), 985-991. https://doi.org/10.1016/j.orgel.2012.02.010.

(4) Cui, L. S.; Nomura, H.; Geng, Y.; Kim, J. U. k.; Nakanotani, H.; Adachi, C. Controlling Singlet-Triplet Energy Splitting for Deep-Blue Thermally Activated Delayed Fluorescence Emitters. Angew. Chemie - Int. Ed. 2017, 56 (6), 1571-1575. https://doi.org/10.1002/anie.201609459.

(5) Rajamalli, P.; Chen, D.; Li, W.; Samuel, I. D. W.; Cordes, D. B.; Slawin, A. M. Z.; ZysmanColman, E. Enhanced Thermally Activated Delayed Fluorescence through Bridge Modification in Sulfone-Based Emitters Employed in Deep Blue Organic LightEmitting Diodes. J. Mater. Chem. C 2019, 7 (22), 6664-6671. https://doi.org/10.1039/c9tc01498e.

(6) Lays, P.; Chen, D.; Rajamalli, P.; Matulaitis, T.; David, B.; Slawin, A. M. Z.; Jacquemin, D.; Zysman-colman, E.; Samuel, I. D. W. The Use of Pyrimidine and Pyrazine Bridges as a Design Strategy to Improve the Performance of Thermally Activated Delayed Fluorescence Organic Light Emitting Diodes. ACS Appl. Mater. Interfaces 2019, 11, 45171-45179. https://doi.org/10.1021/acsami.9b16952.

(7) Dias, F. B.; Penfold, T. J.; Monkman, A. P. Photophysics of Thermally Activated Delayed Fluorescence Molecules. Methods Appl. Fluoresc. 2017, 5 (1), 012001. https://doi.org/10.1088/2050-6120/aa537e.

(8) El-Sayed, M. A. The Triplet State: Its Radiative and Nonradiative Properties. Acc. Chem. Res. 1968, 1 (1), 8-16. https://doi.org/10.1021/ar50001a002.

(9) Liu, M.; Komatsu, R.; Cai, X.; Hotta, K.; Sato, S.; Liu, K.; Chen, D.; Kato, Y.; Sasabe, H.; Ohisa, S.; Suzuri, Y.; Yokoyama, D.; Su, S. J.; Kido, J. Horizontally Orientated Sticklike Emitters: Enhancement of Intrinsic Out-Coupling Factor and Electroluminescence Performance. Chem. Mater. 2017, 29 (20), 8630-8636. https://doi.org/10.1021/acs.chemmater.7b02403.

(10) Yokoyama, D.; Sakaguchi, A.; Suzuki, M.; Adachi, C. Horizontal Orientation of LinearShaped Organic Molecules Having Bulky Substituents in Neat and Doped VacuumDeposited Amorphous Films. Org. Electron. physics, Mater. Appl. 2009, 10 (1), 127137. https://doi.org/10.1016/j.orgel.2008.10.010.

(11) Xiang, S.; Lv, X.; Sun, S.; Zhang, Q.; Huang, Z.; Guo, R.; Gu, H.; Liu, S.; Wang, L. To Improve the Efficiency of Thermally Activated Delayed Fluorescence OLEDs by Controlling the Horizontal Orientation through Optimizing Stereoscopic and Linear Structures of Indolocarbazole Isomers. J. Mater. Chem. C 2018, 6 (21), 5812-5820. https://doi.org/10.1039/c8tc01419a. 
(12) Maeng, J. H.; Ahn, D. H.; Lee, H.; Jung, Y. H.; Karthik, D. Rigid Indolocarbazole Donor Moiety for Highly Efficient Thermally Activated Delayed Fluorescent Device. Dye. Pigment. 2020, 108485. https://doi.org/10.1016/j.dyepig.2020.108485.

(13) Naqvi, B. A.; Schmid, M.; Crovini, E.; Sahay, P.; Naujoks, T.; Rodella, F.; Zhang, Z.; Strohriegl, P.; Bräse, S.; Zysman-Colman, E.; Brütting, W. What Controls the Orientation of TADF Emitters? Front. Chem. 2020, 8 (September), 750. https://doi.org/10.3389/fchem.2020.00750.

(14) Zhang, Z.; Crovini, E.; dos Santos, P. L.; Naqvi, B. A.; Cordes, D. B.; Slawin, A. M. Z.; Sahay, P.; Brütting, W.; Samuel, I. D. W.; Bräse, S.; Zysman-Colman, E. Efficient SkyBlue Organic Light-Emitting Diodes Using a Highly Horizontally Oriented Thermally Activated Delayed Fluorescence Emitter. Adv. Opt. Mater. 2020. https://doi.org/10.1002/adom.202001354.

(15) Cha, J. R.; Lee, C. W.; Lee, J. Y.; Gong, M. S. Design of Ortho-Linkage CarbazoleTriazine Structure for High-Efficiency Blue Thermally Activated Delayed Fluorescent Emitters. Dye. Pigment. 2016, 134, 562-568. https://doi.org/10.1016/j.dyepig.2016.08.023.

(16) Park, H. J.; Han, S. H.; Lee, J. Y. A Directly Coupled Dual Emitting Core Based Molecular Design of Thermally Activated Delayed Fluorescent Emitters. J. Mater. Chem. C 2017, 5 (46), 12143-12150. https://doi.org/10.1039/c7tc03133e.

(17) Yong Joo Cho, Sang Kyu Jeon, Sang-Shin Lee, Eunsun Yu, and J. Y. L. Donor Interlocked Molecular Design for Fluorescence-like Narrow Emission in Deep Blue Thermally Activated Delayed Fluorescent Emitters. Chem.Mater. 2016, 28, 54005405.

(18) Cho, Y. J.; Jeon, S. K.; Chin, B. D.; Yu, E.; Lee, J. Y. The Design of Dual Emitting Cores for Green Thermally Activated Delayed Fluorescent Materials. Angew. Chemie - Int. Ed. 2015, 54 (17), 5201-5204. https://doi.org/10.1002/anie.201412107.

(19) Kim, M.; Jeon, S. K.; Hwang, S. H.; Lee, S. S.; Yu, E.; Lee, J. Y. Highly Efficient and Color Tunable Thermally Activated Delayed Fluorescent Emitters Using a "Twin Emitter" Molecular Design. Chem. Commun. 2016, 52 (2), 339-342. https://doi.org/10.1039/c5cc07999c.

(20) Wei, D.; Ni, F.; Wu, Z.; Zhu, Z.; Zou, Y.; Zheng, K.; Chen, Z.; Ma, D.; Yang, C. Designing Dual Emitting Cores for Highly Efficient Thermally Activated Delayed Fluorescent Emitters. J. Mater. Chem. C 2018, 6 (43), 11615-11621. https://doi.org/10.1039/c8tc02849d.

(21) Chen, D.; Kusakabe, Y.; Ren, Y.; Sun, D.; Wada, Y.; Suzuki, K.; Kaji, H.; Zysman-, E. Multichromophore Molecular Design for Efficient Thermally Activated Delayed Fluorescence Emitters with Near-Unity Photoluminescence Quantum Yields. ChemRxiv 2021. https://doi.org/10.1021/acs.joc.1c01101.

(22) Byeon, S. Y.; Kim, J.; Lee, D. R.; Han, S. H.; Forrest, S. R.; Lee, J. Y. Nearly 100\% Horizontal Dipole Orientation and Upconversion Efficiency in Blue Thermally Activated Delayed Fluorescent Emitters. Adv. Opt. Mater. 2018, 6 (15), 1701340. https://doi.org/10.1002/adom.201701340.

(23) Sharma, N.; Spuling, E.; Mattern, C. M.; Li, W.; Fuhr, O.; Tsuchiya, Y.; Adachi, C.; Bräse, S.; Samuel, I. D. W.; Zysman-Colman, E. Turn on of Sky-Blue Thermally Activated 
Delayed Fluorescence and Circularly Polarized Luminescence (CPL): Via Increased Torsion by a Bulky Carbazolophane Donor. Chem. Sci. 2019, 10 (27), 6689-6696. https://doi.org/10.1039/c9sc01821b.

(24) Kim, M.; Jeon, S. K.; Hwang, S. H.; Lee, S. S.; Yu, E.; Lee, J. Y. Correlation of Molecular Structure with Photophysical Properties and Device Performances of Thermally Activated Delayed Fluorescent Emitters. J. Phys. Chem. C 2016, 120 (5), 2485-2493. https://doi.org/10.1021/acs.jpcc.5b09114.

(25) Schmidt, T. D.; Lampe, T.; Daniel Sylvinson, M. R.; Djurovich, P. I.; Thompson, M. E.; Brütting, W. Emitter Orientation as a Key Parameter in Organic Light-Emitting $\begin{array}{llllll}\text { Diodes. Phys. Rev. Appl. 2017, } 8 \text { (3), } 37001 . & \end{array}$ https://doi.org/10.1103/PhysRevApplied.8.037001.

(26) Senes, A.; Meskers, S. C. J.; Dijkstra, W. M.; Van Franeker, J. J.; Altazin, S.; Wilson, J. S.; Janssen, R. A. J. Transition Dipole Moment Orientation in Films of Solution Processed Fluorescent Oligomers: Investigating the Influence of Molecular Anisotropy. J. Mater. Chem. C 2016, 4 (26), 6302-6308. https://doi.org/10.1039/c5tc03481g.

(27) Senes, A.; Meskers, S. C. J.; Greiner, H.; Suzuki, K.; Kaji, H.; Adachi, C.; Wilson, J. S.; Janssen, R. A. J. Increasing the Horizontal Orientation of Transition Dipole Moments in Solution Processed Small Molecular Emitters. J. Mater. Chem. C 2017, 5 (26), 6555-6562. https://doi.org/10.1039/C7TC01568B.

(28) Gao, Y.; Hlil, A.; Wang, J.; Chen, K.; Hay, A. S. Synthesis of Homo- And Copoly(Arylene Bicarbazole)s via Nucleophilic Substitution Polycondensation Reactions of NH Groups with Activated Dihalides. Macromolecules 2007, 40 (14), 4744-4746. https://doi.org/10.1021/ma0702750.

(29) Zhang, Q.; Zhuang, H.; He, J.; Xia, S.; Li, H.; Li, N.; Xu, Q.; Lu, J. Improved Ternary Memory Performance of Donor-Acceptor Structured Molecules through Cyano Substitution. J. Mater. Chem. C 2015, 3 (26), 6778-6785. https://doi.org/10.1039/c5tc00839e.

(30) Grimme, S. Density Functional Calculations with Configuration Interaction for the Excited States of Molecules. Chem. Phys. Lett. 1996, 259, 128-137.

(31) Santos, P. L.; Ward, J. S.; Data, P.; Batsanov, A. S.; Bryce, M. R.; Dias, F. B.; Monkman, A. P. Engineering the Singlet-Triplet Energy Splitting in a TADF Molecule. J. Mater. Chem. C 2016, 4 (17), 3815-3824. https://doi.org/10.1039/c5tc03849a.

(32) Hosokai, T.; Matsuzaki, H.; Nakanotani, H.; Tokumaru, K.; Tsutsui, T.; Furube, A.; Nasu, K.; Nomura, H.; Yahiro, M.; Adachi, C. By Delocalized Excited States. Sci. Adv. 2017, 3 (May), 1603282. https://doi.org/10.1126/sciadv.1603282.

(33) Noda, H.; Nakanotani, H.; Adachi, C. Excited State Engineering for Efficient Reverse Intersystem Crossing. Sci. Adv. 2018, 4 (6), eaao6910. https://doi.org/10.1126/sciadv.aao6910.

(34) Samanta, P. K.; Kim, D.; Coropceanu, V.; Brédas, J. L. Up-Conversion Intersystem Crossing Rates in Organic Emitters for Thermally Activated Delayed Fluorescence: Impact of the Nature of Singlet vs Triplet Excited States. J. Am. Chem. Soc. 2017, 139 (11), 4042-4051. https://doi.org/10.1021/jacs.6b12124. 
(35) Wada, Y.; Nakagawa, H.; Matsumoto, S.; Wakisaka, Y.; Kaji, H. Molecular Design Realizing Very Fast Reverse Intersystem Crossing in Purely Organic Emitter. ChemRix 2019, No. 2, 1-21. https://doi.org/10.26434/CHEMRXIV.9745289.V1.

(36) Wada, Y.; Nakagawa, H.; Matsumoto, S.; Wakisaka, Y.; Kaji, H. Organic Light Emitters Exhibiting Very Fast Reverse Intersystem Crossing. Nat. Photonics 2020, No. 14, 643-649. https://doi.org/10.1038/s41566-020-0667-0.

(37) Wang, X.; Yan, Q.; Chu, P.; Luo, Y.; Zhang, Z.; Wu, S.; Wang, L.; Zhang, Q. Analysis on Fluorescence of Dual Excitable Eu(TTA)3DPBT in Toluene Solution and PMMA. J. Lumin. 2011, 131 (8), 1719-1723. https://doi.org/10.1016/j.jlumin.2011.03.061.

(38) Crosby, G. A.; Demas, J. N. Measurement of Photoluminescence Quantum Yields. Review. J. Phys. Chem. 1971, 75 (8), 991-1024. https://doi.org/10.1021/j100678a001.

(39) Song, F.; Xu, Z.; Zhang, Q.; Zhao, Z.; Zhang, H.; Zhao, W.; Qiu, Z.; Qi, C.; Zhang, H.; Sung, H. H. Y.; Williams, I. D.; Lam, J. W. Y.; Zhao, Z.; Qin, A.; Ma, D.; Tang, B. Z. Highly Efficient Circularly Polarized Electroluminescence from Aggregation-Induced Emission Luminogens with Amplified Chirality and Delayed Fluorescence. Adv. Funct. Mater. 2018, 28 (17), 1-12. https://doi.org/10.1002/adfm.201800051.

(40) Kumar, M.; Pereira, L. Mixed-Host Systems with a Simple Device Structure for Efficient Solution-Processed Organic Light-Emitting Diodes of a Red-Orange TADF Emitter. ACS Omega 2020, 5 (5), 2196-2204. https://doi.org/10.1021/acsomega.9b03253.

(41) Vecchi, P. A.; Padmaperuma, A. B.; Qiao, H.; Sapochak, L. S.; Burrows, P. E. A Dibenzofuran-Based Host Material for Blue Electrophosphorescence. Org. Lett. 2006, 8 (19), 4211-4214. https://doi.org/10.1021/ol0614121.

\section{TOC Graphic}



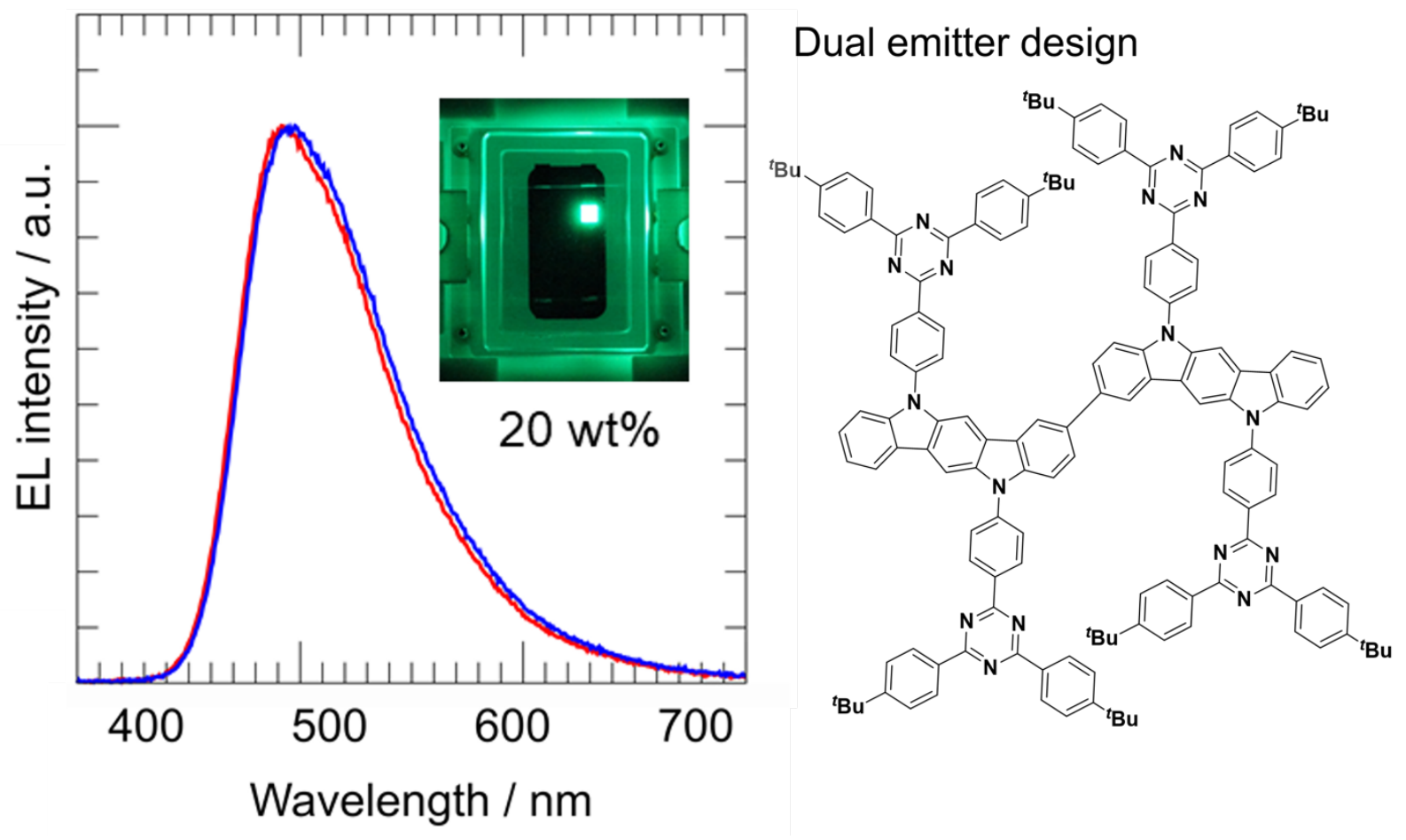